\title{
Quasi-One-Dimensional Metal-Insulator Transitions in Compound Semiconductor Surfaces
}

\author{
J. Z. Zhao, ${ }^{1,2}$ W. Fan, ${ }^{3}$ M. J. Verstraete, ${ }^{4}$ Z. Zanolli,${ }^{5,6}$ J. Fan, ${ }^{1}$ X. B. Yang, ${ }^{7}$ H. Xu, ${ }^{1, *}$ and S. Y. Tong ${ }^{1,8, \dagger}$ \\ ${ }^{1}$ Department of Physics, South University of Science and Technology of China, 518055 Shenzhen, People's Republic of China \\ ${ }^{2}$ Dalian Institute of Chemical Physics, Chinese Academy of Sciences, 116023 Dalian, People's Republic of China \\ ${ }^{3}$ Computational Condensed Matter Physics Laboratory, RIKEN, Wako, Saitama 351-0198, Japan \\ ${ }^{4}$ Département de Physique and European Theoretical Spectroscopy Facility, Universite de Liège, B-4000 Sart Tilman, Liège, Belgium \\ ${ }^{5}$ Peter Grünberg Institute (PGI-1) and Institute for Advanced Simulation (IAS-1), Forschungszebtrum Jülich, D-52425 Jülich, Germany \\ ${ }^{6}$ Institute for Theoretical Solid State Physics and European Theoretical Spectroscopy Facility (ETSF), \\ RWTH Aachen University, D-52056 Aachen, Germany \\ ${ }^{7}$ Department of Physics, South China University of Technology, Guangzhou 510640, People's Republic of China \\ ${ }^{8}$ School of Science and Engineering, The Chinese University of Hong Kong (Shenzhen), 518172 Shenzhen, People's Republic of China
}

(Received 17 January 2016; revised manuscript received 14 April 2016; published 6 September 2016)

Existing examples of Peierls-type 1D systems on surfaces involve depositing metallic overlayers on semiconducting substrates, in particular, at step edges. Here we propose a new class of Peierls system on the $(10 \overline{1} 0)$ surface of metal-anion wurtzite semiconductors. When the anions are bonded to hydrogen or lithium atoms, we obtain rows of threefold coordinated metal atoms that act as one-atom-wide metallic structures. First-principles calculations show that the surface is metallic, and below a certain critical temperature the surface will condense to a semiconducting state. The idea of surface scaffolding is introduced in which the rows are constrained to move along simple up-down and/or sideways displacements, mirroring the paradigm envisioned in Peierls's description. We predict that this type of insulating state should be visible in the partially hydrogenated (1010) surface of many wurtzite compounds.

DOI: 10.1103/PhysRevLett.117.116101

The electronic, vibrational, and magnetic properties of 1D systems are central to current condensed matter physics, from Luttinger liquids to nanotubes and nanowires. Part of the driving force behind this interest is linked to unique properties that 1D systems offer, which may be exploited in nanoscaled electronic and sensing devices. Metallic chains in one dimension, however, are generally unstable and undergo a metal-insulator (MI) phase transition at low temperatures-the so-called Peierls instability $[1,2]$. In the classical Peierls picture, the MI transformation is driven by electronic redistribution via the opening of a band gap at the Fermi level. The Fermi surface nesting features of the original unit cell cause the electronic instability and, consequently, a reduction in total energy. The electronic redistribution of filled states near the Fermi level sets up a charge density wave (CDW) [3-6] responsible for a metal-toinsulator transition. Although the main driving force in a Peierls transition is electronic, the MI transition in real systems is always accompanied by a periodic lattice distortion resulting in a new unit cell due to the strong chargelattice coupling.

CDW in low-dimensional systems deposited on substrates have been reported [7] for quasi-2D systems such as submonolayers of $\mathrm{Pb} / \mathrm{Sn} \mathrm{Ge}(111)$ [8-10] and $\mathrm{In} / \mathrm{Cu}(001)$ [11], and quasi-1D systems such as bands of $\mathrm{In} / \mathrm{Si}(111)$ [12-17], $\mathrm{Au} / \mathrm{Ge}(001)$ [18,19], and $\mathrm{Au} / \mathrm{Si}(553)$ [20,21]. In the quasi-1D systems studied so far, the width $(w)$ of each metal band was at least four to five atoms across. However, later studies on these quasi-2D and -1D systems have called attention to the fact that more complex structural reconstruction forces unrelated to the Peierls transition may be present for all of these cases [22,23]. Indeed, detailed mapping of the Fermi surfaces [24] have revealed a behavior that does not meet the Peierls transition criterion. Effects of atoms from deeper layers are found to be strong on the seemingly 1D systems [25-27]. Further, it has proven very difficult to fabricate, by direct deposition methods, 1D metallic structures which are very narrow (one to two atoms across) and highly uniform. In this perspective, alternative methods to construct wires with sufficiently high length-to-width $(L / w)$ ratio are of great interest and practical importance.

In this Letter, we propose an alternative way to fabricate extremely thin 1D metallic structures with a very high $L / w$ ratio on (1010) surfaces of wurtzite semiconductors. We have carried out systematic studies to determine the feasibility of the idea. We study the electronic, structural, and phonon behaviors of the $\mathrm{GaN}(10 \overline{1} 0)-1 \mathrm{H},-1 \mathrm{Li}$, and $\mathrm{ZnO}(10 \overline{1} 0)-1 \mathrm{H}$, -1Li systems and show that Peierls-type MI transitions indeed occur for these quasi-1D systems. Related results of wurtzite $\mathrm{AlN}(10 \overline{1} 0)-1 \mathrm{H}$ and $\mathrm{BeO}(10 \overline{1} 0)-1 \mathrm{H}$ are briefly shown in the Supplemental Material (SM) [28]. We find that the electronic redistribution and lattice displacement are confined mainly to the rows of surface metal chains, while deeper layer atoms and the surface anions bonded to either $\mathrm{H}$ or $\mathrm{Li}$ serve as supporting scaffolds for the surface metal chains. Depending 
on the "stiffness" of the metal-anion bonds, the MI transition may occur at room temperature or at very low temperatures.

All first-principles calculations are performed using the Vienna $a b$ initio simulation package (VASP) [29,30]. Potentials based on the projector augmented wave method [31] are used to describe ion-electron interactions, and the Perdew and Wang [32] functional is employed. The slab system containing 12 atomic layers separated by $12 \AA$ vacuum regions is found to be numerically well converged. A $k$-point grid of $5 \times 5 \times 3$ is used to sample the bulk Brillouin zone (BZ), while equivalent density grids are applied for the rest of the studied phases. All the structures are relaxed until the forces on each atom are below $0.001 \mathrm{eV} / \AA ̊$. Full phonon dispersion curves are generated with the PHONOPY [33] package.

We start with the wurtzite $(10 \overline{1} 0)$ surface of metal-anion compound semiconductors, where the metals $(M)$ are group II or III atoms, and the anions $(A)$ are either group $\mathrm{V}$ or VI atoms. We focus on two important and representative surfaces: $\mathrm{GaN}(10 \overline{1} 0)$ and $\mathrm{ZnO}(10 \overline{1} 0)$. Large area $\mathrm{GaN}(10 \overline{1} 0)$ and $\mathrm{ZnO}(10 \overline{1} 0)$ surfaces may be grown from suitably chosen substrates or cut and polished from bulk crystals $[34,35]$. We investigate the effect of bonding either hydrogen $(\mathrm{H})$ or lithium $(\mathrm{Li})$ atoms to these surfaces. For $\mathrm{H}$, it is established that the deposited surfaces exhibit two distinct phases [36], a low-temperature $M A-2 \mathrm{H}$ phase where $\mathrm{H}$ bonds to both the surface metal and anion atoms, and a higher-temperature $M A-1 \mathrm{H}$ phase where $\mathrm{H}$ bonds only to the surface anion atoms [see Figs. 1(a) and 1(b)]. It is the latter phase that we are interested in to show Peierls transitions. For $\mathrm{Li}$, we find that the $M A-1 \mathrm{Li}$ phase is the most stable, where the Li atoms bond only to anion sites on the surface [see Fig. 1(c)]. The thermodynamical stability
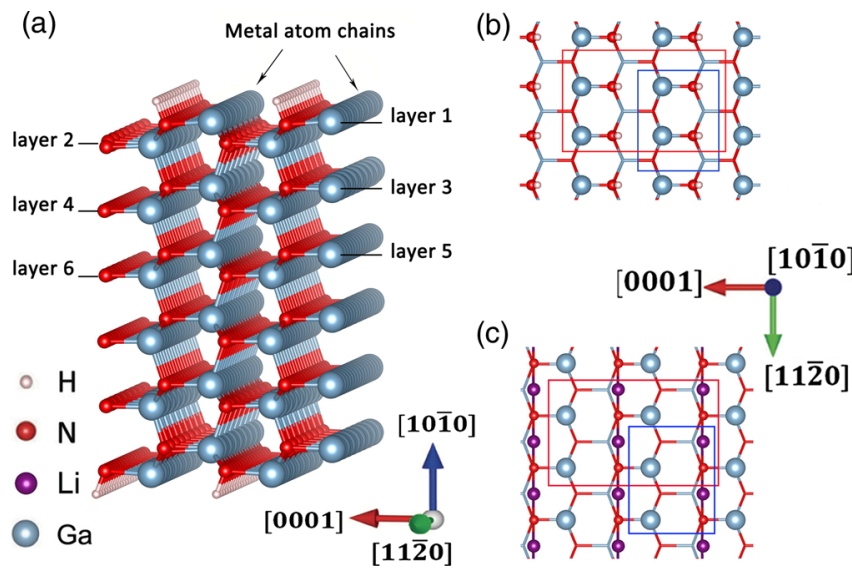

FIG. 1. The structure of $\mathrm{GaN}-1 \mathrm{H}$ and GaN-1Li slabs: Side view (a) of a 12-layer symmetric slab of GaN-1H. Surface Ga forms continuous chains along [1120]. (b) Top view of the $\mathrm{GaN}(10 \overline{1} 0)-1 \mathrm{H}$ and (c) $\mathrm{GaN}(10 \overline{1} 0)-\mathrm{Li}$ surface, in which the surface atoms are shown as spheres. The $p(1 \times 2)$ and the $p(2 \times 2)$ cells are marked by blue and red lines, respectively. The color code to label the atoms is indicated in the figure. of the surfaces is presented in depth in Figs. S1 and S2 of SM [28]. For both the MA-1H and -1Li phase, bulk and surface anion atoms are fourfold coordinated, as are the bulk metal atoms. Only the surface metal atoms are threefold coordinated. The rows of threefold surface metal atoms oriented along the [112̄0] direction (see Fig. 1, blue spheres) run across a full terrace width, broken only at surface defects or step edges. We show below that these atomic chains are prime candidates for demonstrating Peierls-type MI transitions and CDWs. As fabrication and cleaning of these compound semiconductor surfaces are well established, the quasi-1D systems that we propose should be within experimental reach.

Results of $\mathrm{GaN}(10 \overline{1} 0)-1 \mathrm{H}$ are presented in detail here, while similar results of $\mathrm{GaN}(10 \overline{1} 0)-1 \mathrm{Li}, \mathrm{ZnO}(10 \overline{1} 0)-1 \mathrm{H}$, and -1 Li are summarized in Table I with details presented in the Figs. S3 and S4 of SM [28]. For the $-1 \mathrm{H}$ phase, the $p(1 \times 1)$ system is found to be metallic and undergoes a Peierls-type MI transition accompanied by the opening of a band gap. To show this instability, we carry out a phonon frequency analysis. The vibrational dispersion curves [see Fig. 2(a)] show lattice instabilities indicated by imaginary vibrational frequencies in many regions of the BZ. The most unstable frequencies are at the $M$ and $Y$ points. At the $Y$ point, the instability results in an up-down buckling distortion in the surface $\mathrm{Ga}$ chain along the [11 $\overline{2} 0]$ direction. For this mode, the displacements in neighboring chains (in the [0001] direction) are identical, such that the unit cell is $p(1 \times 2)$. At the $M$ point, the unstable mode corresponds to similar up-down displacements along the $[11 \overline{2} 0]$ direction with, in addition, antiphase displacements of the surface $\mathrm{Ga}$ atoms in neighboring chains along the [0001] direction, resulting in a $p(2 \times 2)$ unit cell, which is the most stable configuration.

In the Peierls description, the CDW wave vector must correspond to strong Fermi surface nesting. This nesting behavior is shown in Fig. 2(b) for $\mathrm{GaN}(10 \overline{1} 0)-1 \mathrm{H}$, with two strongly nested surface bands. The nesting vector $q_{1}$

TABLE I. Surface stabilization energy $(\Delta E)$, electronic band gap, and atomic displacement of surface Ga upwards $\left(\Delta d_{u}\right)$ or downwards $\left(\Delta d_{d}\right)$ for a 12-layer slab of wurtzite $\mathrm{GaN}-1 \mathrm{H},-1 \mathrm{Li}$ and $\mathrm{ZnO}-1 \mathrm{H},-1 \mathrm{Li}$. The surface stabilization energy is the energy gained due to surface reconstruction per $p(2 \times 2)$ cell, i.e., the difference between the total energy of the reconstructed surface and the nonreconstructed surface.

\begin{tabular}{lccccc}
\hline \hline & Cell & $\Delta E(\mathrm{eV})$ & $\mathrm{Gap}(\mathrm{eV})$ & $\Delta d_{u}(\AA)$ & $\Delta d_{d}(\AA)$ \\
\hline $\mathrm{GaN}(10 \overline{1} 0)-1 \mathrm{H}$ & $1 \times 2$ & -0.51 & 0.74 & 0.45 & 0.45 \\
& $2 \times 2$ & -0.57 & 0.99 & 0.42 & 0.46 \\
$\mathrm{GaN}(10 \overline{1} 0)-1 \mathrm{Li}$ & $1 \times 2$ & -0.38 & 0.81 & 0.29 & 0.36 \\
& $2 \times 2$ & -0.50 & 1.14 & 0.32 & 0.37 \\
$\mathrm{ZnO}(10 \overline{1} 0)-1 \mathrm{H}$ & $2 \times 2$ & -0.10 & 0.30 & 0.20 & 0.13 \\
$\mathrm{ZnO}(10 \overline{1} 0)-1 \mathrm{Li}$ & $2 \times 2$ & -0.16 & 0.40 & 0.28 & 0.32 \\
\hline \hline
\end{tabular}




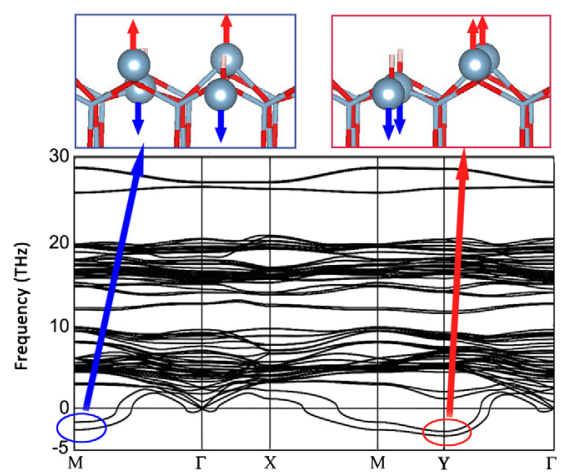

(a)

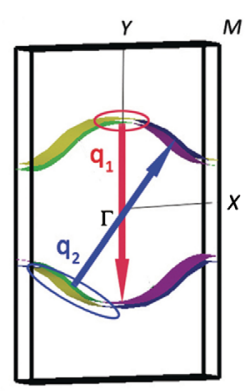

(b)

FIG. 2. (a) Phonon dispersion curves of a $p(1 \times 1)$ 12-layer slab of $\mathrm{GaN}(10 \overline{1} 0)-1 \mathrm{H}$. The phonon frequencies are in units of $\mathrm{THz}$, while the negative values refer to unstable phonon modes. (b) The $2 \mathrm{D}$ Fermi surface of the $p(1 \times 1)$ 12-layer slab of $\mathrm{GaN}(10 \overline{1} 0)-1 \mathrm{H}$. The origin is marked at the center of the $\mathrm{BZ}$ and the nesting vectors are highlighted.

corresponds to the most unstable phonon mode at the $Y$ point, while the nesting vector $q_{2}$ corresponds to the next most unstable phonon mode at the $M$ point. Both nesting vectors nicely fit the nesting condition $2 k_{F}=q_{i}(i=1,2)$. Together, the two nesting vectors induce up-down displacements of the surface $\mathrm{Ga}$ atoms doubling the periodicity along both $[11 \overline{2} 0]$ and [0001] directions and resulting in a $p(1 \times 1)$ to $p(2 \times 2)$ transition.

We next turn to total energy considerations, band gap monitoring, and surface vs bulk contributions in charge redistributions for the transition. First, we expand the surface cell from $p(1 \times 1)$ to $p(1 \times 2)$ and let each atom relax. The system settles into a metastable $p(1 \times 2)$ up-down buckling of surface $\mathrm{Ga}$ atoms, and this reconstruction reduces the total energy by $0.51 \mathrm{eV}$ [compared to the $p(1 \times 1)$ structure] for $\mathrm{GaN}(10 \overline{1} 0)-1 \mathrm{H} \mathrm{eV}$ for $\mathrm{GaN}(10 \overline{1} 0)-1 \mathrm{Li}$ (see Table I). Both systems become semiconducting with the $p(1 \times 2)$ transition having band gaps of 0.74 and $0.81 \mathrm{eV}$, respectively (Table I). Electronic dispersion curves shown in Fig. 3 for $\mathrm{GaN}(10 \overline{10}$ )-1H (with spin polarized bands crossing the Fermi level) and in Fig. S3 of SM [28] for GaN (1010)-1Li indicate that the $p(1 \times 1)$ surface is metallic. If we further expand the surface unit cell, the ground state $p(2 \times 2)$ structure appears for both systems, and the total energy is reduced by 0.57 and $0.50 \mathrm{eV}$ [compared to the $p(1 \times 1)$ structure], while the band gap is widened to 0.99 and $1.14 \mathrm{eV}$, respectively. The splitting of the band in the $p(1 \times 1)$ phase induced by spin polarization also disappears. As the energy gained by the $p(1 \times 1)$ to $p(2 \times 2)$ transition is quite substantial, we expect the $p(2 \times 2)$ phase to be easily detectable below the transition temperature.

These phenomena may be traced back to the nature of the electronic states of the $p(1 \times 1)$ phase (see Fig. S4 of SM [28]). The conduction bands around the Fermi level have three main contributions: from the $4 s, 4 p$ orbitals of

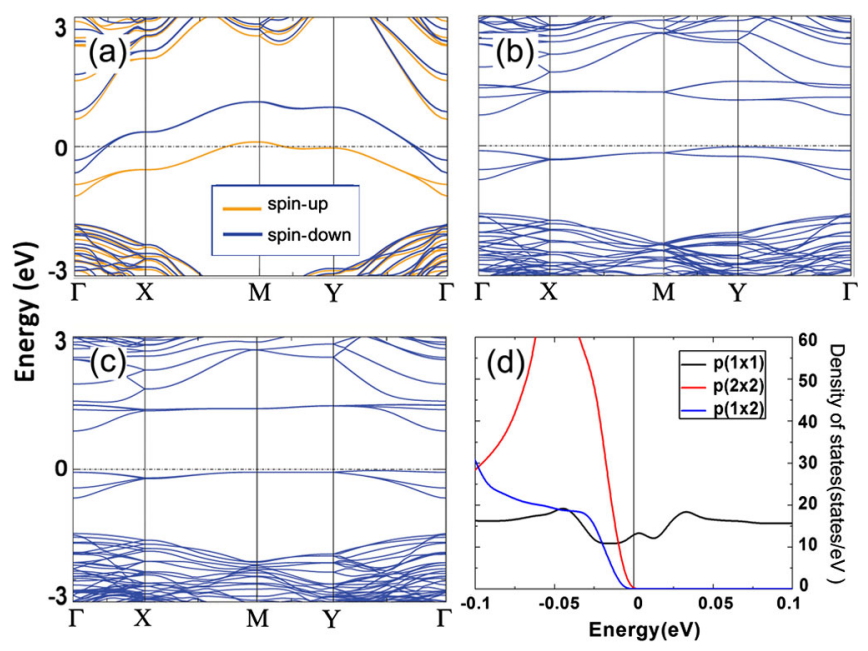

FIG. 3. Band structures of the different phases of $\mathrm{GaN}(10 \overline{1} 0)-1 \mathrm{H}$ in (a) $p(1 \times 1)$, (b) $p(2 \times 2)$, and (c) $p(1 \times 2)$. The Fermi energy is set to zero. The band structures of the $p(1 \times 2)$ and $p(2 \times 2)$ phase are calculated in a $p(2 \times 2)$ cell. Majority and minority spin bands are drawn in different colors in panel (a). The DOS of the three phases are reported in panel (d), the behavior of charge just below the Fermi level suggests that the $p(1 \times 1)$ to $p(1 \times 2)$ transition is of the Peierls type.

surface Ga and $2 p$ orbitals of surface $N$. The orbitals from surface Ga provide strong delocalization along the chain, implying strong charge coupling in the $[11 \overline{2} 0]$ direction. The $\mathrm{N} 2 p$ orbital provides a much weaker link between neighboring chains. Thus, the CDW runs mainly along the chains of surface $\mathrm{Ga}$ atoms. After the surface metal chains are driven into the $p(1 \times 2)$ state, further relaxation into the ground state $p(2 \times 2)$ is caused by conventional energy reduction via lattice relaxation. The density of states (DOS) near the Fermi level $\left(E_{F}\right)$ is the main feature demonstrating that the $p(1 \times 1)$ to $p(1 \times 2)$ transition is pure Peierls type. Comparison of the DOS [Fig. 3(d)] shows that for both systems, charge near $E_{F}$ moves to lower levels as a band gap opens going from the $p(1 \times 1)$ to $p(1 \times 2)$ phase, as expected in a Peierls transition. By contrast, this behavior of the DOS is not seen in the $p(1 \times 2)$ to $p(2 \times 2)$ transition: the charge just below $E_{F}$ does not move to lower levels. This indicates that the total energy gained in the $p(1 \times 2)$ to $p(2 \times 2)$ transition occurs at levels far away from the Fermi energy.

It still remains to show that the CDW and the accompanying lattice distortions are mostly confined to the surface layer, especially along the Ga-atom chains, hence, producing a truly 1D system. For this, we have carried out Bader charge analysis [37-40] of the $p(1 \times 1), p(1 \times 2)$, and $p(2 \times 2)$ phases of the $\mathrm{GaN}(10 \overline{10})-1 \mathrm{H}$ and $-1 \mathrm{Li}$ systems. The charge carried by each atom is listed in Tables SI and SII of SM [28]. For $\mathrm{GaN}(10 \overline{1} 0)-1 \mathrm{H}$ in the $p(1 \times 1)$ phase, each surface $\mathrm{Ga}$ atom carries $+1.0 e$ charge, where $e$ is the absolute value of the charge of an electron. For the $p(1 \times 2)$ phase, the 
surface $\mathrm{Ga}$ that moves up (i.e., away from the bulk) carries a charge of $+0.58 e$ while the neighbor Ga that moves down (i.e., towards the bulk) carries a charge of $+1.44 e$. Going from $p(1 \times 2)$ to $p(2 \times 2)$, the total charge on the atoms remains essentially unchanged. The trend is similar for the $\mathrm{GaN}(10 \overline{1} 0)-1 \mathrm{Li}$ system. These results clearly indicate that the essential charge redistribution occurs mainly within Ga-atom chains along the [1120] direction: the $\mathrm{Ga}$ atoms displaced upwards donate charge to the $\mathrm{Ga}$ atoms displaced downwards. The charge on the inner atomic layer remains practically the same for the three phases. The charge on surface $\mathrm{N}$ or $\mathrm{H} / \mathrm{Li}$ also remains essentially unaffected. The results indicate little tridimensional charge redistribution effects during the MI transition.

Lattice distortions of the $p(1 \times 1)$ to $p(2 \times 2)$ transition as a function of layer (see Fig. S5 of SM [28] for details) show also that buckling is confined mainly to the surface Ga atoms, with up or down displacements of +0.42 and $-0.46 \AA$ from the $p(1 \times 1)$ phase. Such intimate correspondence between charge transfer and lattice distortion is also a hallmark of the Peierls instability.

We carried out similar studies of phonon instability, Fermi surface nesting, electronic DOS behavior, and total energy comparisons for the $\mathrm{GaN}(10 \overline{1} 0)-1 \mathrm{Li}, \mathrm{ZnO}(10 \overline{1} 0)-1 \mathrm{H}$ and $1 \mathrm{Li}, \mathrm{AlN}(10 \overline{1} 0)-1 \mathrm{H}$, and $\mathrm{BeO}(10 \overline{1} 0)-1 \mathrm{H}$ systems. Detailed numbers are presented in Table I and Figs. S6-S8 of SM [28]. The situation for $\mathrm{GaN}(10 \overline{1} 0)-1 \mathrm{Li}$ closely resembles that of $\mathrm{GaN}(10 \overline{1} 0)-1 \mathrm{H}$. For the $\mathrm{ZnO}(10 \overline{1} 0)-1 \mathrm{H}$ and $-1 \mathrm{Li}$ systems, the very small total energy gained by going from $p(1 \times 1)$ to $p(2 \times 2)$ (Table I) suggests that the $p(1 \times 1)$ phase should be stable at room temperature. Indeed, the $p(1 \times 1)$ metallic phase has been observed experimentally for the $\mathrm{ZnO}(10 \overline{1} 0)-1 \mathrm{H}$ system [36]. At low temperatures, we expect these two systems to undergo similar Peierls-type transitions and ultimately condense to the ground state $p(2 \times 2)$. As it is reported in Table SIII of SM [28], the $\mathrm{AlN}(10 \overline{1} 0)-1 \mathrm{H}$ and $\mathrm{BeO}(10 \overline{1} 0)-1 \mathrm{H}$ system undergo another dimerization type with smaller energy reduction and band gap opening, and the $\mathrm{BeO}(10 \overline{1} 0)-1 \mathrm{H}$ surface is $p(1 \times 2)$.

In conclusion, first-principles calculations are used to explore the occurrence of Peierls-type MI transitions and CDW-induced periodic lattice deformations on partially hydrogenated $\mathrm{GaN}(10 \overline{1} 0), \mathrm{ZnO}(10 \overline{1} 0)$, $\mathrm{AlN}(10 \overline{1} 0)$, and $\mathrm{BeO}(10 \overline{1} 0)$ surfaces, as well as partially lithiated $\mathrm{GaN}(10 \overline{1} 0)$ and $\mathrm{ZnO}(10 \overline{1} 0)$ surfaces. The results indicate that the highly symmetric $p(1 \times 1)$ surface is metallic, and condensation to a semiconducting phase is expected. These findings open up the prospect of engineering Peierls-type quasi-1D systems which are just one-atom wide on semiconductors without introducing metallic overlayers. The rows of surface metal atoms are constrained by the scaffold to move along simple up-down distortions that create a CDW below a critical temperature. This combination fulfills the paradigm envisioned in Peierls's original description. The interplay of these CDWs with other degrees of freedom, such as charge and spin in the bulk, may yield distinct features in the spectroscopic or transport properties of such surfaces. Luttinger or Majorana phenomena may appear in the metallic phase. In addition, magnetic dopants in semiconductor surface layers may be coupled by the 1D channels. Our results show a novel pathway for building high $L / w$ ratio $1 \mathrm{D}$ confined nanostructures beyond nanowires, rods, belts, and tubes.

We thank Professor M. H. Whangbo for useful discussions. This work is supported by the National Natural Science Foundation of China (Grants No. 11204185, No. 11334003, and No. 11404159). Z.Z. acknowledges financial support by the EC under the Marie-Curie fellowship (Grant No. PIEF-Ga-2011-300036) and by the Deutsche Forschungsgemeinschaft (German Research Foundation) Grant No. ZA 780/3-1.

*Corresponding author.

xu.h@sustc.edu.cn

Corresponding author.

tong.sy@sustc.edu.cn

[1] R. Peierls, Ann. Physik 395, 1055 (1929).

[2] R. Peierls, Quantum Theory of Solids (Clarendon, Oxford, 1955).

[3] G. Grüner, Density Waves in Solids (Addison-Wesley, Reading, 1994).

[4] R. L. Withers and J. A. Wilson, J. Phys. C 19, 4809 (1986).

[5] M. H. Whangbo and E. Canadell, J. Am. Chem. Soc. 114, 9587 (1992).

[6] S. V. Borisenko, A. A. Kordyuk, A. N. Yaresko, V. B. Zabolotnyy, D. S. Inosov, R. Schuster, B. Büchner, R. Weber, R. Follath, L. Patthey, and H. Berger, Phys. Rev. Lett. 100, 196402 (2008).

[7] P. C. Snijders and H. H. Weitering, Rev. Mod. Phys. 82, 307 (2010).

[8] J. M. Carpinelli, H. H. Weltering, E. W. Plummer, and R. Stumpf, Nature (London) 381, 398 (1996).

[9] J. M. Carpinelli, H. H. Weitering, M. Bartkowiak, R. Stumpf, and E. W. Plummer, Phys. Rev. Lett. 79, 2859 (1997).

[10] J. Avila, A. Mascaraque, E. G. Michel, M. C. Asensio, G. LeLay, J. Ortega, R. Pérez, and F. Flores, Phys. Rev. Lett. 82, 442 (1999).

[11] T. Nakagawa, G. I. Boishin, H. Fujioka, H. W. Yeom, I. Matsuda, N. Takagi, M. Nishijima, and T. Aruga, Phys. Rev. Lett. 86, 854 (2001).

[12] H. W. Yeom, S. Takeda, E. Rotenberg, I. Matsuda, K. Horikoshi, J. Schaefer, C. M. Lee, S. D. Kevan, T. Ohta, T. Nagao, and S. Hasegawa, Phys. Rev. Lett. 82, 4898 (1999).

[13] J. R. Ahn, J. H. Byun, H. Koh, E. Rotenberg, S. D. Kevan, and H. W. Yeom, Phys. Rev. Lett. 93, 106401 (2004).

[14] S. J. Park, H. W. Yeom, S. H. Min, D. H. Park, and I. W. Lyo, Phys. Rev. Lett. 93, 106402 (2004).

[15] C. González, F. Flores, and J. Ortega, Phys. Rev. Lett. 96, 136101 (2006). 
[16] A. A. Stekolnikov, K. Seino, F. Bechstedt, S. Wippermann, W. G. Schmidt, A. Calzolari, and M. Buongiorno Nardelli, Phys. Rev. Lett. 98, 026105 (2007).

[17] H.-J. Kim and J.-H. Cho, Phys. Rev. Lett. 110, 116801 (2013).

[18] J. Schäfer, C. Blumenstein, S. Meyer, M. Wisniewski, and R. Claessen, Phys. Rev. Lett. 101, 236802 (2008).

[19] S. Meyer, J. Schäfer, C. Blumenstein, P. Höpfner, A. Bostwick, J. L. McChesney, E. Rotenberg, and R. Claessen, Phys. Rev. B 83, 121411 (2011).

[20] J. R. Ahn, P. G. Kang, K. D. Ryang, and H. W. Yeom, Phys. Rev. Lett. 95, 196402 (2005).

[21] P. C. Snijders, S. Rogge, and H. H. Weitering, Phys. Rev. Lett. 96, 076801 (2006).

[22] A. Mascaraque, J. Avila, J. Alvarez, M. C. Asensio, S. Ferrer, and E. G. Michel, Phys. Rev. Lett. 82, 2524 (1999).

[23] J. N. Crain, A. Kirakosian, K. N. Altmann, C. Bromberger, S. C. Erwin, J. L. McChesney, J.-L. Lin, and F. J. Himpsel, Phys. Rev. Lett. 90, 176805 (2003).

[24] M. D. Johannes and I. I. Mazin, Phys. Rev. B 77, 165135 (2008).

[25] C. Blumenstein, J. Schäfer, M. Morresi, S. Mietke, R. Matzdorf, and R. Claessen, Phys. Rev. Lett. 107, 165702 (2011).

[26] Y. J. Sun, S. Agario, S. Souma, K. Sugawara, Y. Tago, T. Sato, and T. Takahashi, Phys. Rev. B 77, 125115 (2008).

[27] J. Aulbach, J. Schäfer, S. C. Erwin, S. Meyer, C. Loho, J. Settelein, and R. Claessen, Phys. Rev. Lett. 111, 137203 (2013).
[28] See Supplemental Material http://link.aps.org/supplemental/ 10.1103/PhysRevLett.117.116101, for more discussions on experimental feasibility and electronic properties.

[29] G. Kresse and J. Furthmüller, Comput. Mater. Sci. 6, 15 (1996).

[30] G. Kresse and J. Hafner, J. Phys. Condens. Matter 6, 8245 (1994).

[31] G. Kresse and J. Furthmüller, Phys. Rev. B 54, 11169 (1996).

[32] J. P. Perdew and Y. Wang, Phys. Rev. B 45, 13244 (1992).

[33] A. Togo, F. Oba, and I. Tanaka, Phys. Rev. B 78, 134106 (2008).

[34] K. Fujito, S. Kubo, and I. Fujimura, MRS Bull. 34, 313 (2009).

[35] J. J. Chen, X. R. Deng, and H. Deng, J. Mater. Sci. 48, 532 (2013).

[36] Y. Wang, B. Meyer, X. Yin, M. Kunat, D. Langenberg, F. Traeger, A. Birkner, and C. Wöll, Phys. Rev. Lett. 95, 266104 (2005).

[37] W. Tang, E. Sanville, and G. Henkelman, J. Phys. Condens. Matter 21, 084204 (2009).

[38] E. Sanville, S. D. Kenny, R. Smith, and G. Henkelman, J. Comput. Chem. 28, 899 (2007).

[39] G. Henkelman, A. Arnaldsson, and H. Jónsson, Comput. Mater. Sci. 36, 354 (2006).

[40] M. Yu and D. R. Trinkle, J. Chem. Phys. 134, 064111 (2011). 